\title{
Growth of Prussian blue microcubes under a hydro-thermal condition: a possible non-classical crystallization by a mesoscale self-assembly
}

Xiao-Jun Zheng, Qin Kuang, Tao Xu, Zhi-Yuan Jiang, Shu-Hong Zhang, Zhao-Xiong

$$
\text { Xie*, Rong-Bing Huang, Lan-Sun Zheng.. }
$$

State Key Laboratory for Physical Chemistry of Solid Surfaces, Department of Chemistry, College of Chemistry and Chemical Engineering, Xiamen University, Xiamen 361005, China.

\section{Contents}

1. HRTEM image of the nanocubes of the $\mathbf{4 0}$ min products.

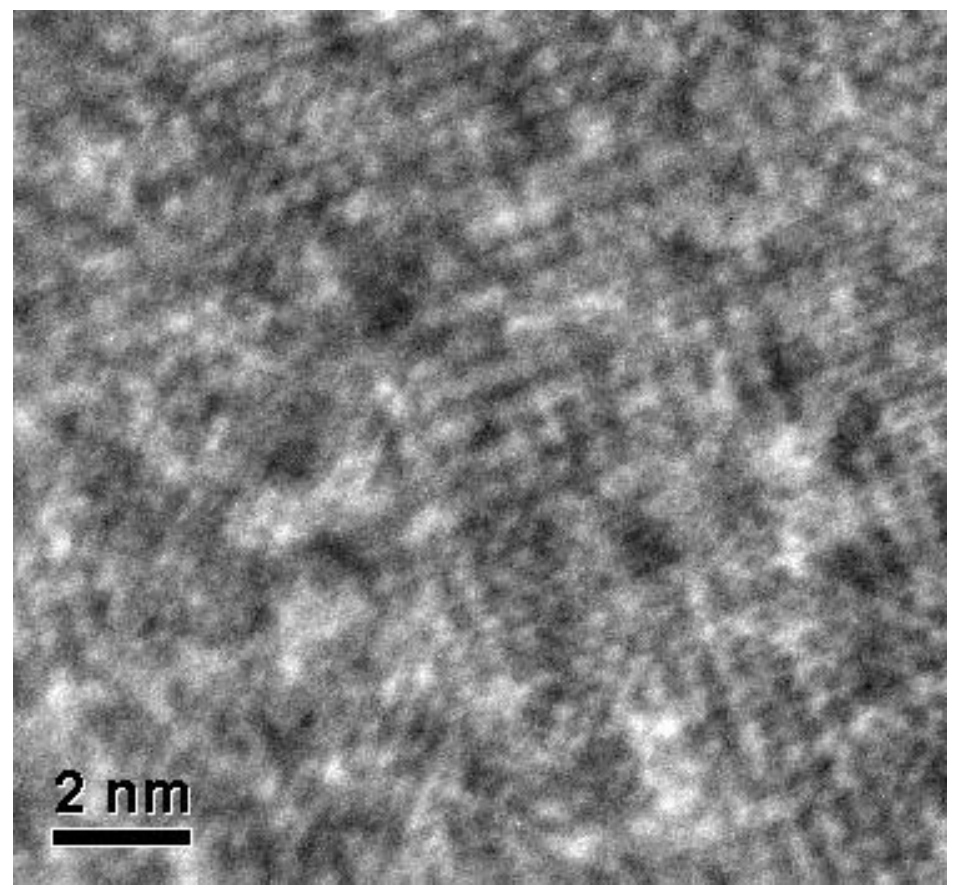

